\title{
High fluid shear stress prevents atherosclerotic plaque formation by promoting endothelium denudation and synthetic phenotype of vascular smooth muscle cells
}

\author{
JUAN WANG $^{1}$, YAN WANG ${ }^{1}$, LIN SHENG $^{1}$, TIAN HE $^{2}$, XIANG NIN $^{1}$, \\ AIYING XUE ${ }^{1}$, HUA ZHANG ${ }^{3}$ and ZHENDONG LIU $^{3}$ \\ ${ }^{1}$ Department of Cardiology, The Second Hospital of Shandong University, Jinan, Shandong 250033; \\ ${ }^{2}$ Department of Anesthesiology, Shandong Provincial Hospital Affiliated to Shandong First Medical University, \\ Jinan, Shandong 250021; ${ }^{3}$ Cardio-Cerebrovascular Control and Research Center, Basic Medical \\ College, Shandong First Medical University, Jinan, Shandong 250062, P.R. China
}

Received September 22, 2020; Accepted March 25, 2021

DOI: $10.3892 / \mathrm{mmr} .2021 .12216$

\begin{abstract}
Low blood fluid shear stress (SS) promotes vascular remodeling and atherosclerosis; however, the effects of high (H)SS on vascular remodeling and atherogenesis is not fully clarified. The major goal of this study was to investigate the role of HSS in atherosclerotic plaque formation. A perivascular SS modifier was implanted in the right carotid artery of apolipoprotein $\mathrm{E}(\mathrm{ApoE})^{-/-}$mice to induce HSS, whereas the left carotid artery represented undisturbed (U)SS as a control in vivo. In vitro modeling used human umbilical vein endothelial cells and vascular smooth muscle cells exposed to HSS (2.5 Pa) using a parallel-plate flow system. The results demonstrated that there were no plaque formations or endothelial cells in the $\mathrm{HSS}$ regions of the carotid artery in $\mathrm{ApoE}^{-/-}$mice. The number of umbilical vein endothelial cells was markedly decreased in a time-dependent manner in HSS. HSS significantly decreased $\alpha$-smooth muscle actin and increased osteopontin protein expression levels compared with USS in vascular
\end{abstract}

Correspondence to: Professor Zhendong Liu, Cardio-Cerebrovascular Control and Research Center, Basic Medical College, Shandong First Medical University, 18877 Jingshi Road, Jinan, Shandong 250062, P.R. China

E-mail: zhendongliu876@126.com

Dr Juan Wang, Department of Cardiology, The Second Hospital of Shandong University, 247 Beiyuan Street, Jinan, Shandong 250033, P.R. China

E-mail: moonlikewang@163.com

Abbreviations: ApoE, apolipoprotein E; EC, endothelial cell; ES, endostatin; HSS, high shear stress; HUVEC, human umbilical vein endothelial cell; MMP, matrix metalloproteinase; SS, shear stress; LSS, low shear stress; USS, undisturbed shear stress; VSMC, vascular smooth muscle cell

Key words: SS, atherosclerotic plaque, endothelium denudation, phenotypic conversion, smooth muscle cell smooth muscle cells $(\mathrm{P}<0.05)$. In addition, HSS significantly increased the protein expression levels of collagen $\alpha 1$ (XVIII) chain/endostatin and matrix metalloproteinase- 8 in vascular smooth muscle cells. These data indicated that HSS may prevent atherosclerotic plaque formation through endothelium denudation and contractile-to-synthetic phenotypic conversion of smooth muscle cells.

\section{Introduction}

Vascular wall shear stress (SS) is crucially important in regulating vascular physiology and pathobiology of the vessel wall in atherosclerosis (1). Low (L)SS promotes vascular inflammation and atherosclerosis, whereas high $(\mathrm{H}) \mathrm{SS}$ is protective (2-7). Although there have been a number of studies on endothelial cell (EC) responses in LSS environments, little attention has been devoted to understanding the role of HSS in atherosclerosis or atherosclerotic plaque formation (8).

HSS, usually regarded as $>2.5 \mathrm{~Pa}$, has been reported to contribute to adaptive outward remodeling, pathological remodeling leading to saccular aneurysm initiation and instability of atherosclerotic plaques (9). Cheng et al (10) developed a perivascular SS modifier, referred to as a cast, that induced changes in SS patterns in apolipoprotein E (ApoE)-deficient mice. They found that atherosclerotic lesions developed in the LSS region, whereas no atherosclerotic lesions were found in the HSS region.

Vascular ECs are exposed to hemodynamic forces under normal physiological conditions. A previous study demonstrated that early-stage atherosclerosis is characterized by endothelial dysfunction (11). ECs are sensors of blood fluid $\mathrm{SS}$ and transduce the frictional force from blood flow into biochemical signals that regulate gene expression and cell behavior through specialized pathways, such as caveolae or caveolin-1 (8). Endothelial dysfunction in atherosclerosis impairs endothelium-dependent vasodilation and may lead to other pathophysiological consequences, such as increased chemotactic and adhesion molecule expression (12), increased monocyte/macrophage recruitment and accumulation, 
decreased EC regeneration, and increased vascular smooth muscle cells (VSMCs) proliferation and migration. However, it is unknown whether VSMC phenotype affects EC properties during vascular remodeling and endothelium recovery. The aim of the present study was to investigate the effects of HSS on ECs and VSMCs and to explore the potential mechanism of HSS on the vessel remodeling.

\section{Materials and methods}

Animal models. Male ApoE ${ }^{-/-}$mice $(n=40$; age 8 weeks; weight, 25-30 g) were obtained from the Key Laboratory of Cardiovascular Remodeling and Function Research of Shandong University. Among them, 15 mice were used for histological section, 16 mice were used for molecular biological detection and nine mice were used for Oil Red of vessel. The specific-pathogen free mice were housed at a constant temperature $\left(24^{\circ} \mathrm{C}\right)$ and humidity (50-60\%), fed a high-fat Western-type diet containing $0.25 \%$ cholesterol and provided water ad libitum. All experiments were performed in compliance with the Guide for the Care and Use of Laboratory Animals published by the US National Institutes of Health (NIH Publication No. 85-23, revised 1985) and Shandong University. The experimental protocol was approved by the institutional animal ethics committee at the Second Hospital of Shandong University [Shandong, China; KYLL-2019(KJ)P-0161].

SS modifier placement. A cast was used to induce standardized changes in SS in vivo as previously described $(10,13)$. Mice were anesthetized by intraperitoneal injection of $0.08 \%$ sodium pentobarbital $(40 \mathrm{mg} / \mathrm{kg})$ prior to SS modifier placement surgery. The cast was implanted in the right carotid artery of $\mathrm{ApoE}^{-/-}$mice and imposed a fixed geometry on the vessel wall that caused gradual stenosis. Consequently, the blood flow in the vessel segment inside the cast increased and SS was elevated (HSS region). Conversely, the blood flow upstream from the cast decreased and SS was lowered (LSS region). The blood flow in a straight segment of the contralateral left carotid artery without cast was undisturbed SS (USS region); this served as a control.

Tissue harvesting. To compare the effects of the three types of SS on the carotid lesion, 40 mice were humanely killed at 10 weeks after cast implantation. The mice were deeply anesthetized with $5.0 \%$ sevoflurane and then euthanized by exsanguination by aspirating the blood from the left ventricle. After collecting blood through heart puncture, a reservoir perfusion apparatus that allowed direct manipulation of perfusion pressure was used to perfuse the vessel. Vessels were perfused with PBS at a constant physiological pressure of $100 \mathrm{mmHg}$, then constant pressure perfusion in situ with $4 \%$ polyformaldehyde. The bilateral common carotid arteries were carefully removed and fixed them in $4 \%$ polyformaldehyde overnight at $4^{\circ} \mathrm{C}$. After carefully removing the cast, the three different SS regions of each mouse were classified, and the tissues $(n=15)$ were embedded in paraffin compound for serial cryosections at room temperature.

Human umbilical vein endothelial cells (HUVECs) in HSS. An in vitro HSS model was established HUVECs [purchased from the American Type Culture Collection (ATCC)] in endothelial cell medium containing 5\% FBS (cat. no. 1001; Sciencell Research Laboratories, Inc.), as previous described (13-15). Controlled with USS, HSS (2.5 Pa) was imposed to HUVECs for 1-2 h using a parallel-plate flow system after HUVECs were cultured up to the fourth passage with $1 \times 10^{6}$ per plate. The viability of HUVECs was assessed using an MTT assay (Sigma-Aldrich; Merck KGaA; M2128-1G) with DMSO for dissolving crystals and a microplate reader at the wavelength $570 \mathrm{~nm}$, according to the manufacturer's instructions.

Histological and immunofluorescence staining. Serial cryosections $(6 \mu \mathrm{m})$ were stained with hematoxylin and eosin (Harris $5 \mathrm{~g} / \mathrm{l}$; Sigma-Aldrich; Merck KGaA) at room temperature for $30 \mathrm{~min}$. Consecutive sections were stained with Sirius red (1.0 g/l; Beyotime Institute of biotechnology) for collagen and muscle fibers were stained using Masson Trichrome staining solution (10 g/l; Sigma-Aldrich; Merck KGaA) at room temperature for $60 \mathrm{~min}$. Oil-red O (5.0 g/l; Sigma-Aldrich; Merck KGaA) staining was used to identify lipid-rich lesions at room temperature for $30 \mathrm{~min}$. Aortic arches and carotid arteries were stained with Oil Red $\mathrm{O}$ for atherosclerotic lesions in the LSS region. The stained cryosections were imaged and analyzed using the Image-Pro Plus 6.0 automated image analysis system (Media Cybernetics, Inc.) attached to a color CCD video camera (fluorescent; x50-1,000; Olympus BX51; Olympus Corporation). Intima was defined as the area bounded by the endothelium and the internal elastic lamina and media were defined as the area bounded by the internal and external elastic laminae (16).

Tissue sections were also used for immunofluorescence staining using antibodies against mouse CD31 (1:500; cat. no. sc-376764; Santa Cruz Biotechnology, Inc.), collagen $\alpha 1$ (XVIII) chain (COL18A1; 1:500; cat. no. ab275746; Abcam), matrix metalloproteinase (MMP-8; 1:500; cat. no. PA5-79687; Invitrogen; Thermo Fisher Scientific, Inc.) overnight at $4^{\circ} \mathrm{C}$. The secondary antibodies were Alexa 488-conjugated donkey anti-rabbit IgG (1:2,000; cat. no. A32790; Invitrogen; Thermo Fisher Scientific, Inc.) and Alexa 568-conjugated donkey anti-goat IgG (1:2,000; cat. no. A11057; Invitrogen; Thermo Fisher Scientific, Inc.). Images were acquired using an LSM710 laser scanning confocal microscope (Zeiss AG).

Following HSS exposure, cultured HUVECs were fixed with $4 \%$ paraformaldehyde and probed with rabbit anti-CD31 (1:1,000 dilution; cat. no. sc-376764; Santa Cruz Biotechnology, Inc.), and VSMCs (ATCC) with rabbit anti-COL18A1 (1:500 dilution; cat. no. ab275746; Abcam), MMP-8 (1:800 dilution; cat. no. PA5-79687; Invitrogen; Thermo Fisher Scientific, Inc.) overnight at $4^{\circ} \mathrm{C}$. VSMCs were cultured with the supernatant of HUVECs. Then, they were stained with FITC-conjugated goat anti-rabbit IgG (1:200; cat. no. TA130021; OriGene Technologies, Inc.) polyclonal antibodies for $30 \mathrm{~min}$ at $37^{\circ} \mathrm{C}$. Counterstaining for the nuclei was performed by incubating with DAPI (cat. no. D3571; Invitrogen; Thermo Fisher Scientific, Inc.) for $5 \mathrm{~min}$ at room temperature. Images were acquired with a laser scanning confocal microscope.

Western blot assay. Proteins were extracted from $50 \mathrm{mg}$ carotid artery specimens of LSS, HSS and USS regions 
A

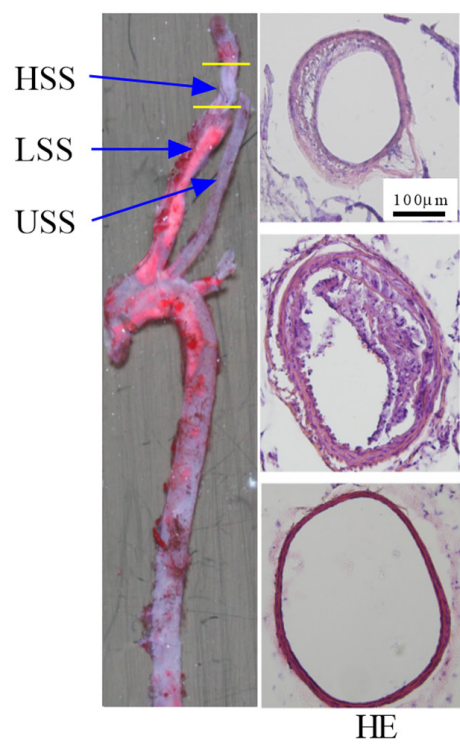

B

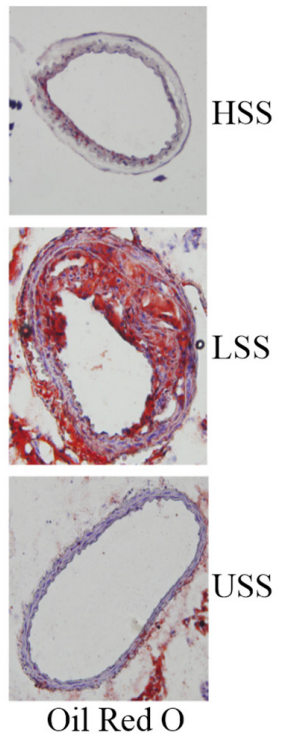

C

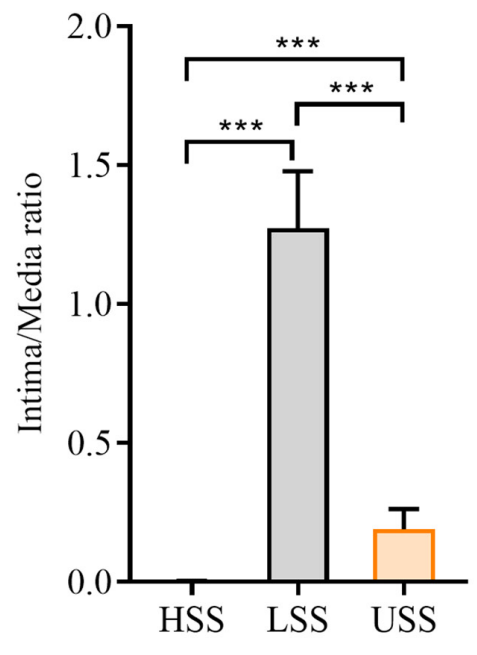

Figure 1. Arterioscleroticplaque formation in different carotid artery regions. (A) Aortic arches and carotid arteries were stained with Oil Red O for atherosclerotic lesions; images from the LSS region. Yellow lines demarcate the position of the cast with the HSS region; upstream from the cast is the LSS region, and the contralateral carotid artery is the USS region. (B) Representative images are presented from the HSS, LSS and USS regions stained with HE. Scale bar, $100 \mu \mathrm{m}$. (C) Quantification of the intima-media ratio obtained by measuring the area confined by the external elastic lamina of the cast-implanted carotid artery in different regions. Values are expressed as the mean \pm standard deviation. ${ }^{*}{ }^{* *} \mathrm{P}<0.001$. HE, hematoxylin and eosin; HSS, high shear stress; LSS low shear stress; USS, undisturbed shear stress.

using lysis buffer (cat. no. P0013; Beyotime Institute of Biotechnology). Equal amounts of protein $(20 \mu \mathrm{l})$ were separated on $10 \%$ SDS-PAGE and transferred to a nitrocellulose membrane (Bio-Rad Laboratories, Inc.). After being blocked with $5 \%$ non-fat milk for $2 \mathrm{~h}$ at room temperature, the blots were washed in TBS $+0.05 \%$ Tween-20 (TBS-T) three times for $10 \mathrm{~min}$ each and incubated at $4^{\circ} \mathrm{C}$ overnight with an appropriate primary antibody: Rabbit anti- $\beta$-actin (1:500; cat. no. AF5006; Beyotime Institute of Biotechnology), rabbit anti-CD31 (1:1,000; cat. no. sc-376764; Santa Cruz Biotechnology, Inc.), rabbit anti- $\alpha$-smooth muscle actin (SMA; 1:1,000; cat. no. 144-60525-100; RayBiotech, Inc.), rabbit anti-osteopontin (OPN; 1:500; cat. no. 119-10057; RayBiotech, Inc.), rabbit anti-COL18A1 (1:500; cat. no. ab275746; Abcam), rabbit anti-MMP8 (1:1,000; cat. no. PA5-79687; Invitrogen; Thermo Fisher Scientific, Inc.). Following incubation, the blots were washed with TBS-T and incubated with horseradish peroxidase-conjugated secondary antibody (1:5,000; cat. no. sc-2357; Santa Cruz Biotechnology, Inc.) for $2 \mathrm{~h}$ at room temperature. The membranes were washed three times with TBS-T, and protein expression were visualized by Digital Gel Imaging system (AlphaEaseF ${ }^{\text {TM }}$ software; serial 2200; ProteinSimple) with enhanced chemiluminescence detection reagents (Merk KGaA).

ELISA. HSS was used on HUVECs for $2 \mathrm{~h}$. The supernatant obtained from HUVECs with different shear stress (USS, HSS, LSS) was harvested for culturing the VSMCs. The concentrations of endostatin (ES; a fragment of COL18A1) and MMP-8 were determined by using commercially available ELISA kits (cat. no. ab-281282; Abcam) following the manufacturer's protocol. Either a monoclonal antibody of ES or MMP-8 rom the kit was added to the assay diluents $(100 \mu \mathrm{l})$. The plates were incubated for $2 \mathrm{~h}$ at $37^{\circ} \mathrm{C}$. Each well was washed three times with wash buffer. The substrate solution $(200 \mu \mathrm{l})$ was added for $30 \mathrm{~min}$ to stop the reaction. The optical density was determined with a microplate reader at the wavelength $450 \mathrm{~nm}$.

Statistical analysis. The experiments were repeated three times. SPSS version 16.0 (SPSS Inc.) was used for statistical analysis. Data are expressed as the mean \pm standard deviation. Statistical evaluation was carried out by two-tailed unpaired Student's t-test between two groups or by one-way ANOVA with Bonferroni's post hoc test among three groups. $\mathrm{P}<0.05$ was considered to indicate a statistically significant difference.

\section{Results}

HSS reduces atherosclerotic plaque formation in Apo $E^{-1-}$ mice. Plaque formation was assessed by $\mathrm{HE}$ and Oil Red $\mathrm{O}$ staining (Fig. 1A). No plaque formations were detected in the HSS and USS regions, whereas plaque formation and fat content were observed in the LSS region in all $15 \mathrm{ApoE}^{-/-}$mice (Fig. 1A and B). The intima-media ratio in the HSS region was significantly lower compared with the USS region, and decreased to near zero $(\mathrm{P}<0.001$; Fig. $1 \mathrm{C})$. The ratio in the LSS region was significantly higher compared with that in the USS or HSS regions (both $\mathrm{P}<0.05$ ).

HSS attenuates the vascular ECs and CD31 expression in mice. CD31, also known as platelet endothelial cell adhesion molecule, has been used as an EC marker. Compared with the normal expression of CD31 in USS regions with intact endometrium, the expression levels of CD31 were increased in LSS (Fig. 2A). Compared with the normal expression of ECs in the LSS and USS regions, there were fewer ECs, or almost no ECs 
A

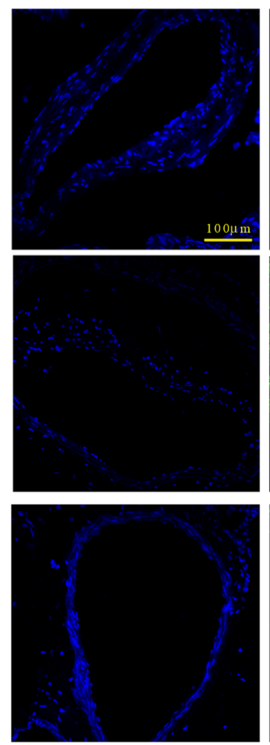

DAPI
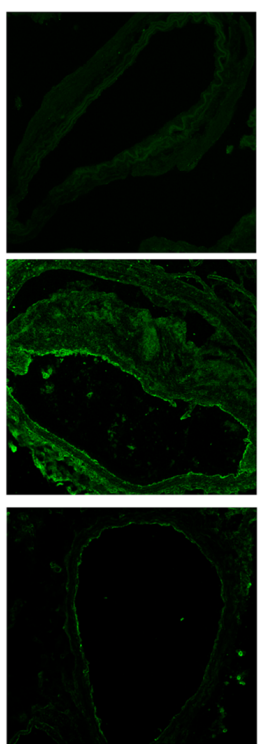

CD31

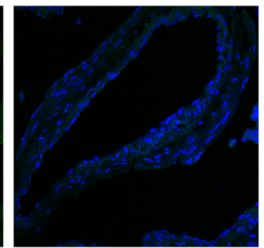

HSS
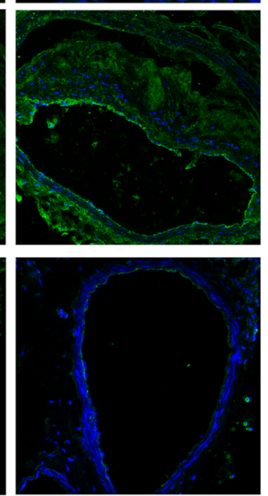

Merged
B
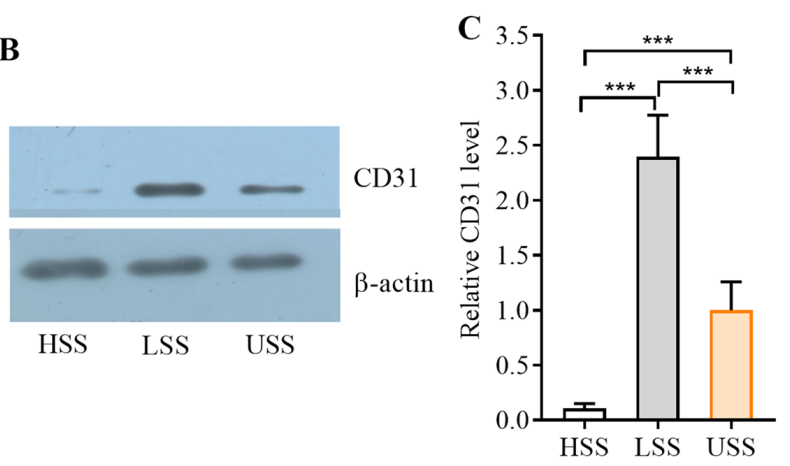

Figure 2. Vascular CD31 protein expression levels of different regions in carotid artery regions. (A) Representative immunofluorescence images acquired by a laser scanning confocal microscopy demonstrating CD31 staining of the endothelium in the HSS, LSS and USS regions; the cell nuclei were stained with DAPI. Scale bar, $100 \mu \mathrm{m}$. (B) Representative western blots and $(\mathrm{C})$ semi-quantification of relative $\mathrm{CD} 31$ protein expression levels in the different carotid artery regions; $\beta$-actin was used a loading control. Values are expressed as the mean \pm standard deviation. ${ }^{* * *} \mathrm{P}<0.001$. HSS, high shear stress; LSS low shear stress; USS, undisturbed shear stress.

detected by immunofluorescence in the HSS regions (Fig. 2A). CD31 protein expression levels were assessed by western blotting and were significantly decreased in the HSS region and significantly increased in the LSS region compared to the USS region $(\mathrm{P}<0.001$; Fig. 2B and $\mathrm{C})$.

HSS decreases the number of HUVECs in a time-dependent manner. HUVECs were stimulated by HSS for 0,1 and $2 \mathrm{~h}$. The CD31 expression in intensity over time was detected via western blotting using equal numbers of ECs, while EC numbers were counted. CD31 immunofluorescence and MTT demonstrated that the counts of HUVECs were markedly decreased in a time-dependent manner (Fig. 3A and B, respectively). CD31 protein expression levels in ECs were assessed using western blotting after the HUVECs were stimulated by HSS for $2 \mathrm{~h}$; no significant difference was identified ( $\mathrm{P}>0.05$; Fig. $3 \mathrm{C}$ and $\mathrm{D}$ ).

Collagen content and synthetic phenotype of VSMCs are enhanced by HSS. Given the crucial role in atherogenesis,

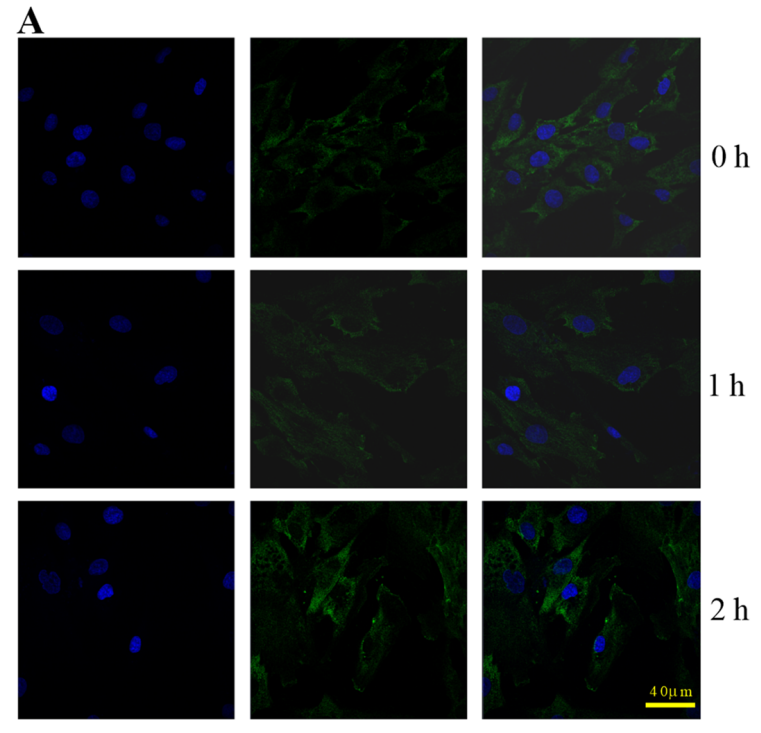

B

CD31

Merged

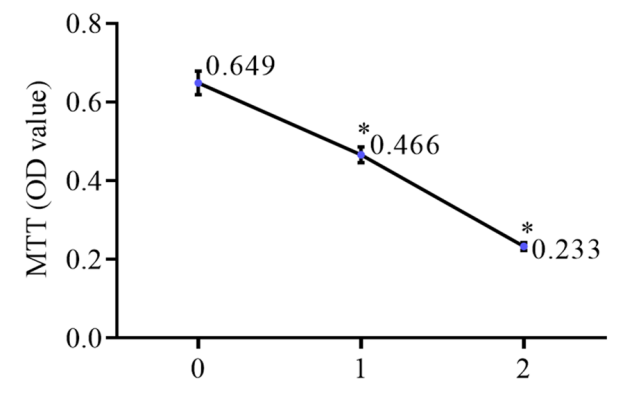

Time (h)

C
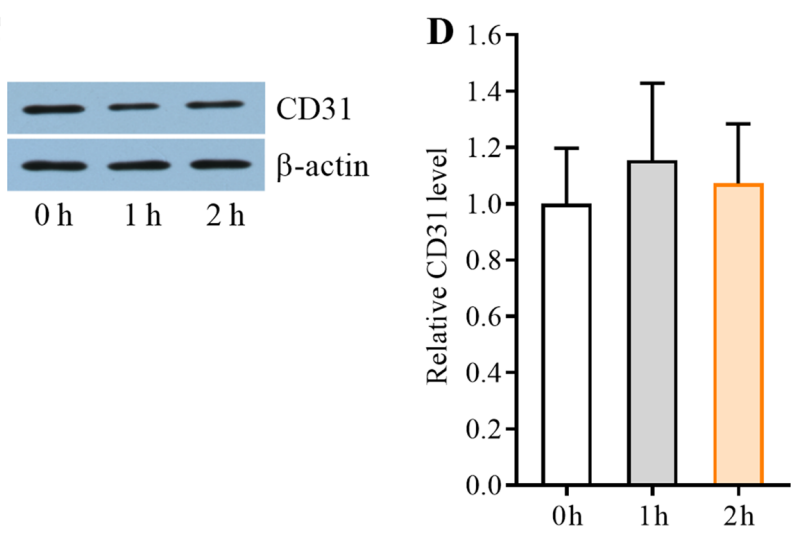

Figure 3. Time-dependent CD31 expression and cell viability after high shear stress. (A) Representative immunofluorescence images obtained by laser scanning microscopy show CD31 staining in HUVECs after HSS for 0, 1 and $2 \mathrm{~h}$; nuclei were stained with DAPI. Magnification, $\mathrm{x} 40$. (B) Changes in the viability of HUVECs at different times were analyzed by MTT. (C) Representative western blots and (D) semi-quantification of relative CD31 protein expression levels at the different time points of HSS; $\beta$-actin was used a loading control. Data are expressed as the mean \pm standard deviation. ${ }^{*} \mathrm{P}<0.05$ vs. 0 h. OD, optical density.

collagen content and VSMC phenotype were examined in the HSS, LSS and USS regions. The collagen contents and VSMCs were determined using Sirius red and Masson's Trichrome staining. Collagen content was increased and the VSMC content were decreased in the HSS region compared with the USS region, although the differences were not significant $(\mathrm{P}>0.05$; Fig. 4A-C). To explore the effects of HSS on the VSMC 
A
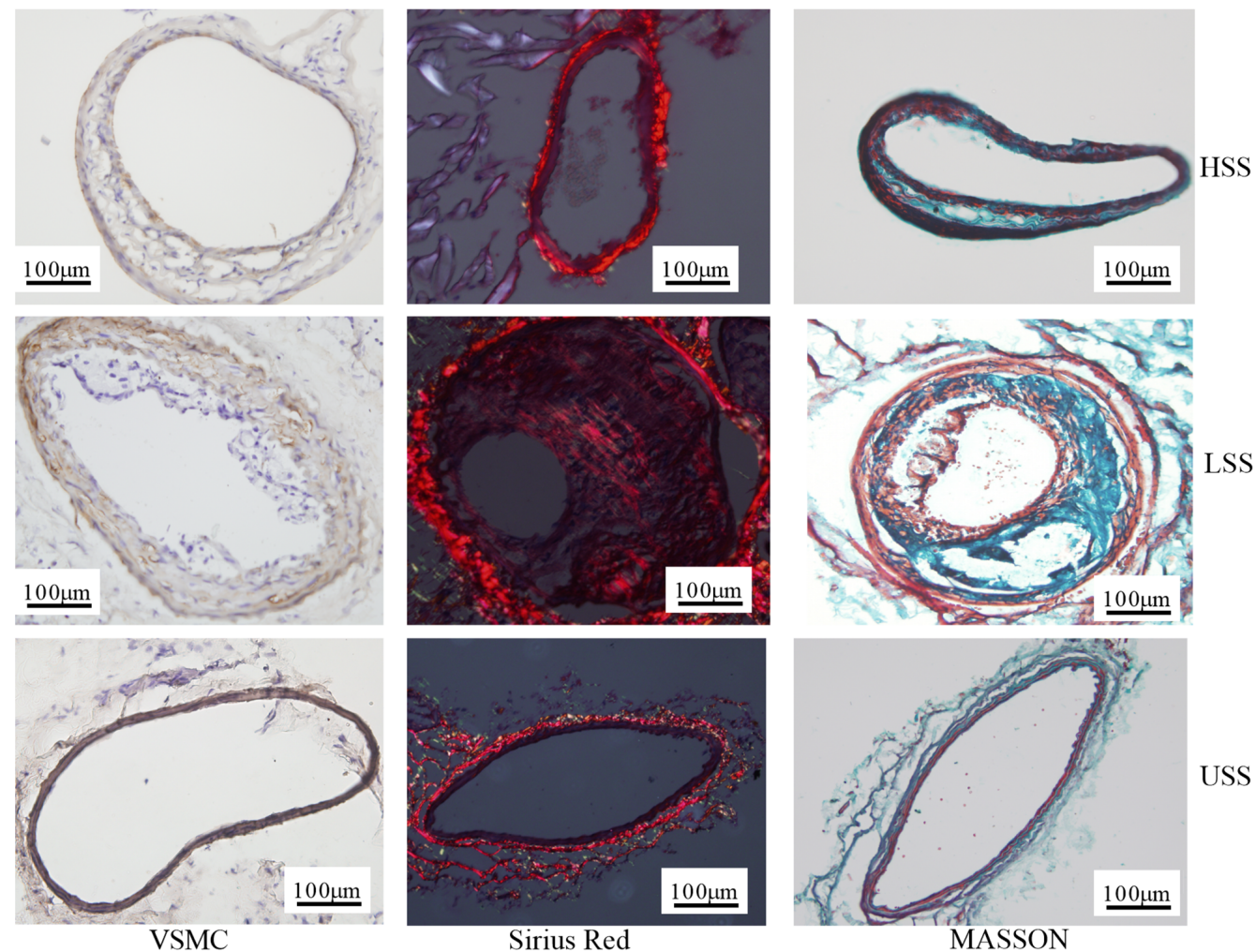

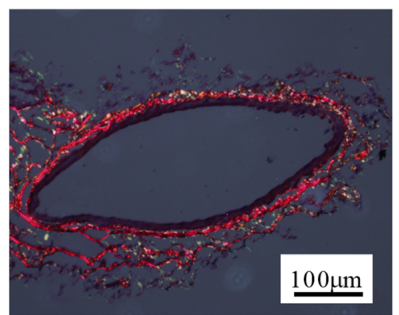

Sirius Red
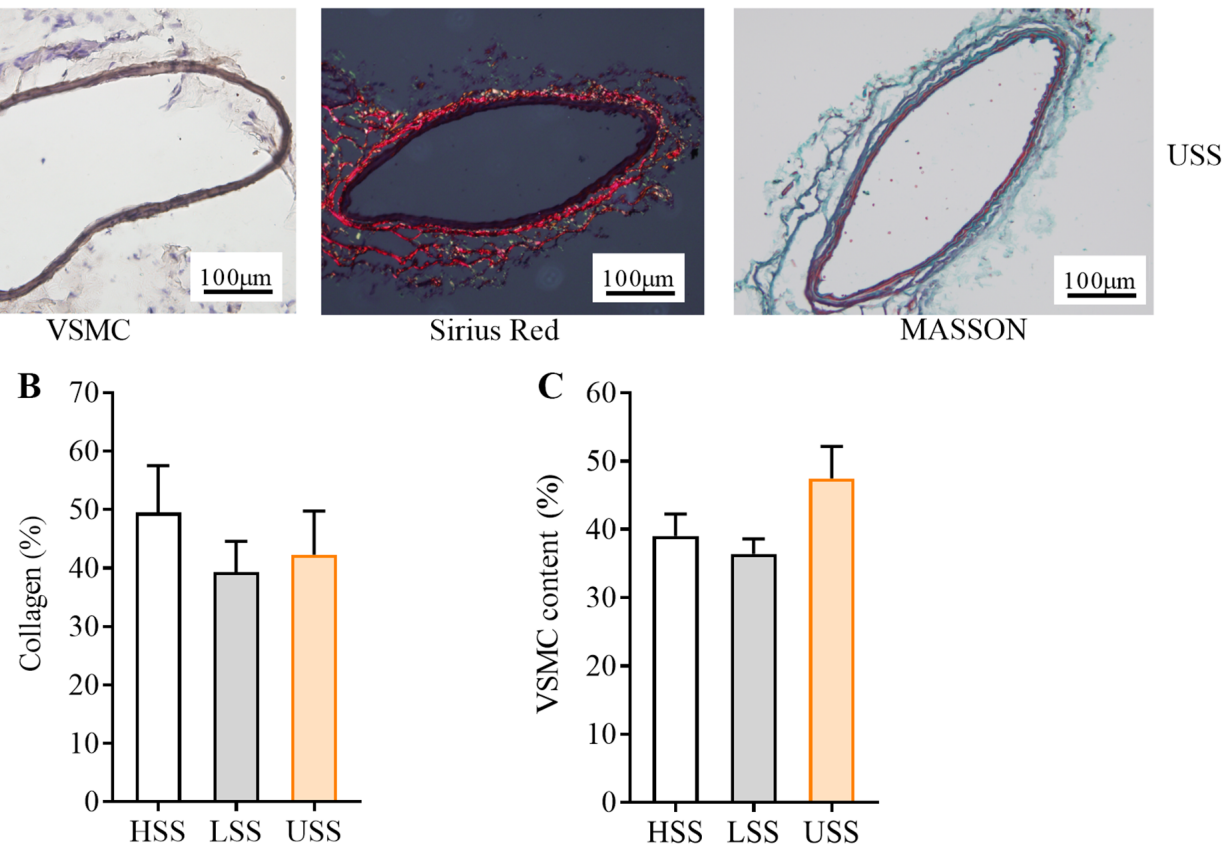

D
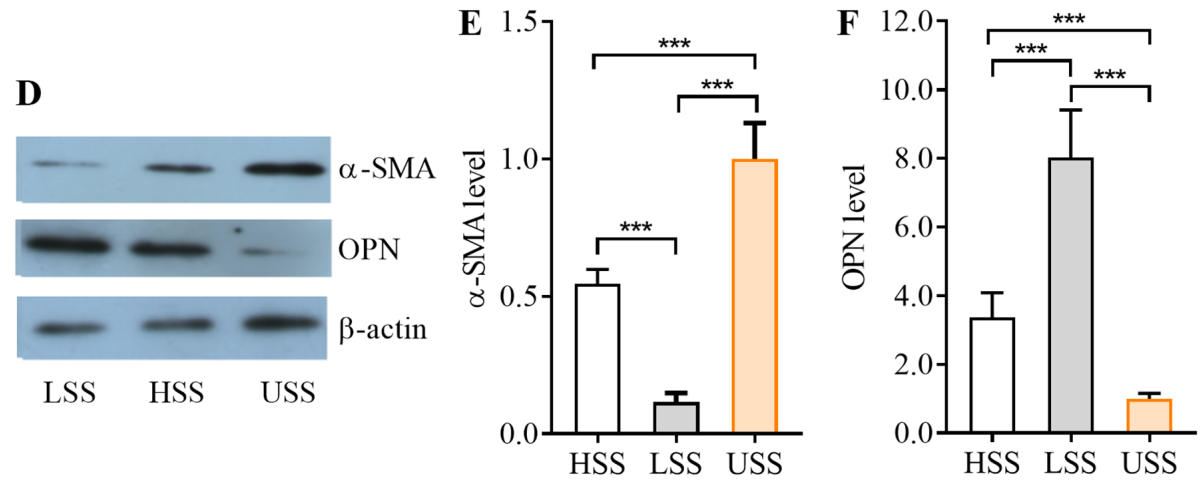

Figure 4. Contents and phenotype of VSMCs and collagen in different SS regions. (A) Representative images of carotid artery sections immunostained with $\alpha$-SMA, or stained with sirius red or Masson's trichrome compound solution for VSMC and collagen in HSS, LSS and USS regions. Scale bar, $100 \mu \mathrm{m}$. Histograms showed the quantification of (B) collagen and (C) VSMCs content (\%). Data are expressed as the mean \pm standard deviation; $\mathrm{n}=15$. (D) Representative western blots and semi-quantification of (E) $\alpha$-SMA protein expression levels, as an indicator for SMC contractile phenotype, and (F) OPN protein expression levels, as an indicator for SMC synthetic phenotype in the three regions; $\beta$-actin was used as a loading control. Data are expressed as the mean \pm standard deviation, $\mathrm{n}=16 .{ }^{* * *} \mathrm{P}<0.001$. $\alpha$-SMA, $\alpha$-smooth muscle actin; HSS, high shear stress; LSS low shear stress; OPN, osteopontin; SS, shear stress; USS, undisturbed shear stress; VSMC, vascular smooth muscle cell.

phenotype, the expression of $\alpha$-SMA and OPN, which are widely used to determine VSMC phenotype $(17,18)$, were examined by western blotting. Fig. 4D and F showed the $\alpha$-SMA expression in contractile phenotype of VSMCs, and OPN expression in 
A
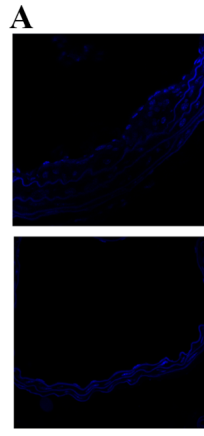

DAPI
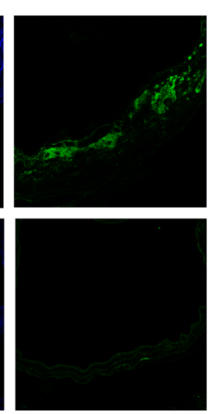

COL $18 \mathrm{Al}$

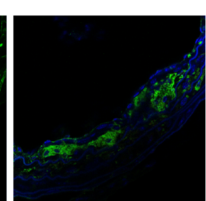
HSS

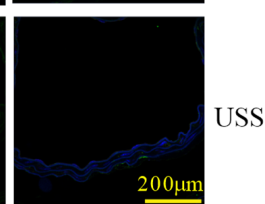

Merged

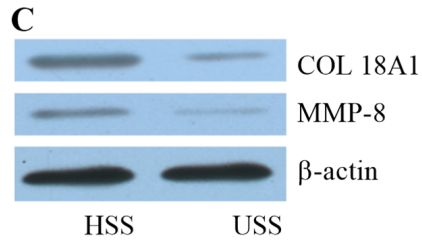

D

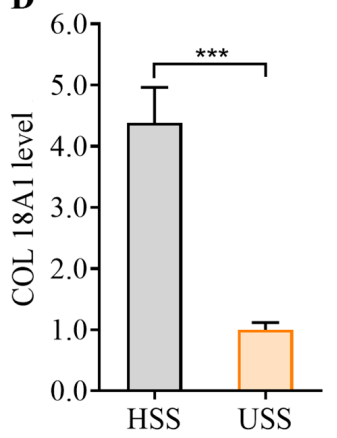

B
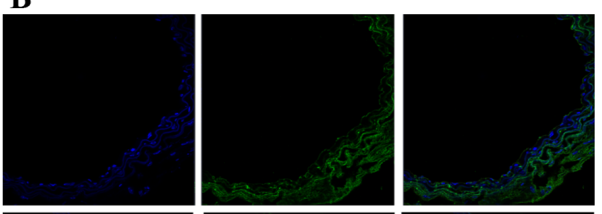

HSS
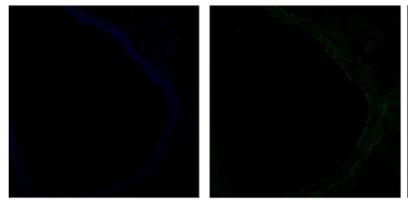

MMP-8

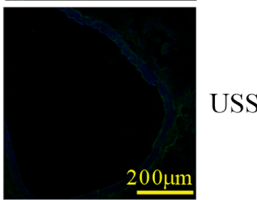

Merged

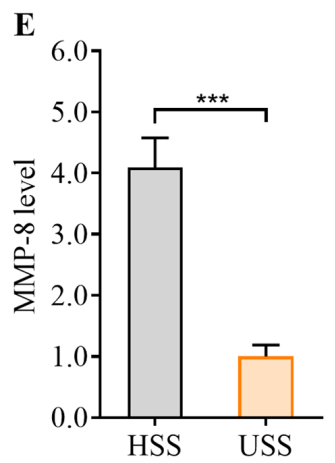

Figure 5. Vascular COL18A1 and MMP-8 protein expression levels of HSS compared with USS regions in carotid arteries. Representative immunofluorescence images showing (A) COL18A1 staining and (B) MMP-8 staining; nuclei were stained with DAPI. Scale bar, $200 \mu \mathrm{m}$. (C) Representative western blots and semi-quantification of (D) COL18A1 and (E) MMP-8 protein expression levels; $\beta$-actin was used as a loading control. Data were obtained from a pool of 16 animals. Data are expressed as the mean \pm standard deviation. ${ }^{* * *} \mathrm{P}<0.001$. COL18A1, collagen $\alpha 1$ (XVIII) chain; HSS, high shear stress; MMP, matrix metalloproteinase; USS, undisturbed shear stress.

synthetic phenotype of VSMCs. $\alpha$-SMA protein expression level was significantly lower and OPN expression was higher in the HSS region compared with expression in the USS region (both $\mathrm{P}<0.001$; Fig. 4D-F). It indicated that HSS could induce the conversion of VSMCs from the contractile phenotype ( $\alpha$-SMA) to synthetic phenotype (OPN).

HSS promotes the expression of vascular COL18A1 and $M M P-8$ in mouse carotid arteries. To verify the activity of synthetic VSMCs induced by HSS, the protein expression levels of COL18A1 and MMP-8 in carotid arteries were determined using immunofluorescence (Fig. 5A and B) and western blotting (Fig. 5C). The expression levels of COL18A1 and MMP-8 were significantly higher in the HSS region compared with the USS region, as detected by western blotting $(\mathrm{P}<0.001$; Fig. 5D and E).

HSS induces higher levels of COL18A1/ES and MMP-8 in VSMCs. To further confirm the activity of synthetic VSMCs, VSMCs were exposed to HSS for $2 \mathrm{~h}$ and the expression levels of COL18A1 and MMP-8 were detected using immunofluorescence and the supernatant concentrations of ES and MMP-8 were measured using ELISA. ES is a fragment of COL18A1 and regarded as a possible surrogate marker for subclinical atherosclerosis with basement membrane degradation $(19,20)$. The expression levels of COL18A1 and MMP-8 in the VSMCs were increased by HSS (Fig. 6A and B). The supernatant concentrations of COL18A1 and MMP-8 were significantly higher after HSS $2 \mathrm{~h}$ compared with USS $(\mathrm{P}<0.001$; Fig. 6C and D).

\section{Discussion}

Physiological laminar SS serves a crucial role in vascular function (1). To investigate the possible role of HSS on vascular remodeling, EC function and VSMC phenotype conversion induced by HSS was determined. The major findings of the present study were that HSS i) reduced/inhibited carotid plaque formation in $\mathrm{ApoE}^{-/}$mice; ii) decreased EC counts and attenuated CD31 expression; and iii) induced the conversion of VSMCs from the contractile phenotype to synthetic phenotype.

In this study, no atherosclerotic plaque and low intima-media ratio were found in the HSS region as well as in the USS region in the carotid artery of $\mathrm{ApoE}^{-/-}$mice. These data indicated that HSS may prevent atherosclerotic plaque formation. In addition, experimental results showed that ECs were not detected and the expression of CD31 was low in the HSS region of the carotid artery of $\mathrm{ApoE}^{-/-}$mice. However, in vitro experiments showed that HSS did not induce changes in CD31 expression of HUVECs, although the number of HUVECs was significantly decreased by HSS. These results, at least to some extent, coincide with previous studies $(4,21,22)$. Thus, it was suggested that preventing atherosclerotic plaque formation by HSS resulted from endothelial denudation rather than the conversion of the ECs phenotype. Lipid infiltration or deposition, which is a distinguishing feature of atherosclerosis and atherosclerotic plaque, is closely related to endothelial dysfunction (2). After endothelial denudation, the EC monolayer is removed and the subintimal lipid-binding may be prevent (23). Finally, 
A
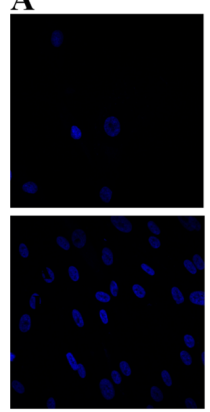

DAPI
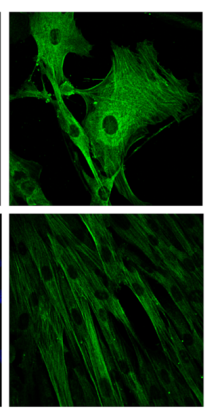

COL 18Al

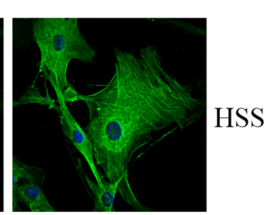

B
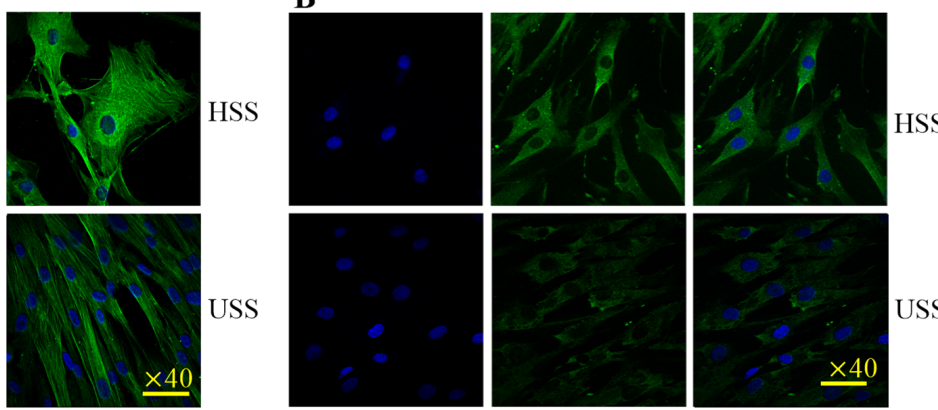

Merged

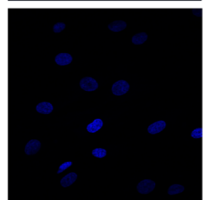

DAPI

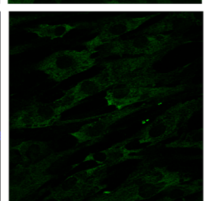

MMP-8

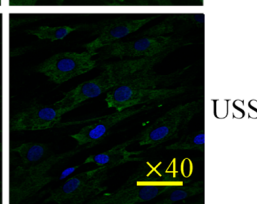

Merged
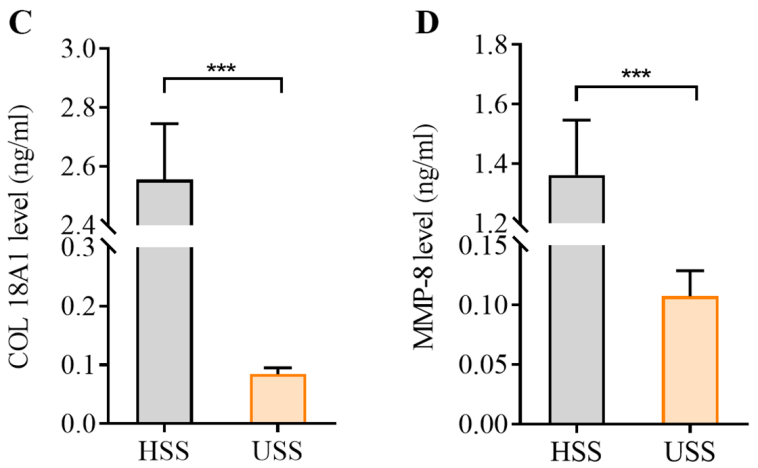

Figure 6. Relative levels of COL18A1 and MMP-8 after HSS in VSMCs. Representative immunofluorescence images showing (A) COL18A1 and (B) MMP-8 staining after VSMCs were exposed to HSS for $2 \mathrm{~h}$. Magnification, $\mathrm{x} 40$. (C) Levels of COL18A1 (ng/ml) and (D) MMP-8 in VSMC supernatant were detected by ELISA. Data are expressed as the mean \pm standard deviation. ${ }^{* * *} \mathrm{P}<0.001$. COL18A1, collagen $\alpha 1$ (XVIII) chain; HSS, high shear stress; MMP, matrix metalloproteinase; USS, undisturbed shear stress.

the formation of atherosclerosis and atherosclerotic plaque is limited.

Conversely, results from the present study found that LSS increased CD31 expression, which was consistent with our previous study (13). Results from the previous study demonstrated that CD31 may be a major mechanoreceptor of LSS for activation of JNK followed by $N F-\kappa B$ and vascular cell adhesion protein 1 (13). LSS is known to increase EC inflammatory response, proliferation and apoptosis leading to the occurrence and development of atherosclerosis. CD31, also called platelet endothelial cell adhesion molecule1, is one of important member of the immunoglobulin superfamily (13). Thus, LSS may induce the upregulation of CD31 expression in $\mathrm{ECs}$.

VSMCs and ECs are in close contacted with each other in blood vessels (24). VSMCs could be modulated by the ECs, which are directly exposed to the blood flow. Normal laminar SS modulate neointima accumulation (25). However, HSS was previously demonstrated to promote certain cellular responses, such as metalloproteinase activity to degrade matrix components, VSMC apoptosis and reduced matrix synthesis (26). In the present study, $\alpha$-SMA expression was found to be significantly decreased whereas OPN expression was remarkably increased by HSS compared with USS. It indicated that HSS may induce VSMCs to convert from a contractile to a synthetic phenotype.

In the present study, the secretory activity of VSMCs was activated by HSS. Moreover, the concentrations of COL18A1/ES and MMP-8 were significantly elevated by HSS. ES is universally regarded as a circulating angiostatic factor derived from the cleavage of COL18A1 (27-29) and has dramatic effects on EC gene expression as well as the capacity to inhibit EC migration, proliferation and survival (29-33). Elevated circulating ES closely correlates with adverse pulmonary hemodynamics in pulmonary arterial hypertension $(28,34)$. COL18A1/ES expression was reported to be significantly increased in the intima and media of remodeled vessels (34). MMP-8 expression is correlated with atherosclerotic lesion formation and progression (35-38). A previous study demonstrated that the knockdown of MMP-8 in HUVECs results in a decrease in capillary-like network formation, cell proliferation and migration, and impaired the capacity of in vivo angiogenesis (38). Elevated ES levels may result from vasculature basement membrane degradation (39).

In the present study, a cast was used to induce HSS, which may have limited carotid vasodilative activity. This might be the reason why there was no carotid artery lumen enlargement, aneurysm or dissection in $\mathrm{ApoE}^{-/-}$mice. However, the results are consistent with previous studies showing that HSS and elevated COl18A1/ES are risk factors for artery aneurysm and dissection with pathological increscent of synthetic phenotype VSMCs (40-42), which showed the same results of pathologic intimal denudation in arterial vessels. A major limitation to the present study is that the potential pathways of COL18A1/ES and MMP-8 in EC denudation and VSMC phenotypic conversion induced by HSS were not investigated. In addition, the apoptosis of ECs stimulated by HSS has not been discussed in the current study. These questions will be the focus of further research.

In summary, the results of the present study indicated that HSS may prevent atherosclerotic plaque formation. The probable mechanism is that HSS may induce endothelium denudation and contractile-to-synthetic phenotypic conversion 
of VSMCs. An improved understanding of the underlying mechanisms of SS in vessel remodeling would potentially lead to improving strategies for atherosclerotic disease management and novel targets for pharmacological intervention.

\section{Acknowledgements}

Not applicable.

\section{Funding}

This work was supported by The National Natural Science Foundation of China (grant nos. 81500232, 81670432 and 81973139); The Key Technology Research and Development Project of Shandong Province (grant nos. 2017GSF18169 and 2018GSF118044); The Shandong Provincial Natural Science Foundation (grant nos. ZR2020MH047 and ZR2017PH050). Funding bodies did not have any role in the design or performance of the study, nor in the interpretation of the results or writing of the manuscript.

\section{Availability of data and materials}

The datasets used and/or analyzed during the current study are available from the corresponding author on reasonable request.

\section{Authors' contributions}

JW and ZL confirm the authenticity of all the raw data, and designed and conducted the study. JW, YW, LS, TH, XN, AX, HZ and ZL collected and analyzed the data. JW, HZ and ZL interpreted the data and wrote the manuscript. All authors read and approved the final manuscript.

\section{Ethics approval and consent to participate}

Not applicable.

\section{Patient consent to publication}

Not applicable.

\section{Competing interests}

The authors declare that they have not competing interests.

\section{References}

1. Cunningham KS and Gotlieb AI: The role of shear stress in the pathogenesis of atherosclerosis. Lab Invest 85: 9-23, 2005.

2. Gijsen F, van der Giessen A, van der Steen A and Wentzel J: Shear stress and advanced atherosclerosis in human coronary arteries. J Biomech 46: 240-247, 2013.

3. Luong L, Duckles H, Schenkel T, Mahmoud M, Tremoleda JL, Wylezinska-Arridge M, Ali M, Bowden NP, Villa-Uriol MC, van der Heiden $\mathrm{K}$, et al: Heart rate reduction with ivabradine promotes shear stress-dependent anti-inflammatory mechanisms in arteries. Thromb Haemost 116: 181-190, 2016.

4. Malek AM, Alper SL and Izumo S: Hemodynamic shear stress and its role in atherosclerosis. JAMA 282: 2035-2042, 1999.

5. Liu Z, Zhao Y, Wang X, Zhang H, Cui Y, Diao Y, Xiu J, Sun X and Jiang G: Low carotid artery wall shear stress is independently associated with brain white-matter hyperintensities and cognitive impairment in older patients. Atherosclerosis 247: 78-86, 2016.
6. Chen Y, Yu H, Zhu J, Zhang H, Zhao Y, Dong Y, Cui Y, Gong G, Chai Q, Guo Y and Liu Z: Low carotid endothelial shear stress associated with cerebral vessel disease in an older population: A subgroup analysis of a population-based prospective cohort study. Atherosclerosis 288: 42-50, 2019.

7. Zhao Y, Dong Y, Wang J, Sheng L, Chai Q, Zhang H and Liu Z: Longitudinal association of carotid endothelial shear stress with renal function decline in aging adults with normal renal function: A population-based cohort study. Sci Rep 9: 2051, 2019.

8. Brown AJ, Teng Z, Evans PC, Gillar JH, Samady H and Bennett MR: Role of biomechanical forces in the natural history of coronary atherosclerosis. Nat Rev Cardiol 13: 210-220, 2016.

9. Dolan JM, Kolega J and Meng H: High wall shear stress and spatial gradients in vascular pathology: A review. Ann Biomed Eng 41: 1411-1427, 2013.

10. Cheng C, Tempel D, van Haperen R, van der Baan A, Grosveld F, Daemen MJ, Krams R and de Croom R: Atherosclerotic lesion size and vulnerability are determined by patterns of fluid shear stress. Circulation 113: 2744-2753, 2006.

11. Chiu JJ and Chien S: Effects of disturbed flow on vascular endothelium: Pathophysiological basis and clinical perspectives. Physiol Rev 91: 327-387, 2011.

12. Zakkar M, Chaudhury H, Sandvik G, Enesa K, Luong LA, Cann S, Mason JC, Krams R, Clark AR, Haskard DO and Evans PC: Increased endothelial mitogen-activated protein kinase phosphatase-1 expression suppresses proinflammatory activation at sites that are resistant to atherosclerosis. Circ Res 103: 726-732, 2008.

13. Wang J, An FS, Zhang W, Gong L, Wei SJ, Qin WD, Wang XP, Zhao YX, Zhang Y, Zhang C and Zhang MX: Inhibition of c-Jun $\mathrm{N}$-terminal kinase attenuates low shear stress-induced atherogenesis in apolipoprotein E-deficient mice. Mol Med 17: 990-999, 2011.

14. Namdee K, Carrasco-Teja M, Fish MB, Charoenphol P and Eniola-Adefeso O: Effect of variation in hemorheology between human and animal blood on the binding efficacy of vascular-targeted carriers. Sci Rep 5: 11631, 2015.

15. Moriguchi T and Sumpio BE: PECAM-1 phosphorylation and tissue factor expression in HUVECs exposed to uniform and disturbed pulsatile flow and chemical stimuli. J Vasc Surg 61: 481-488, 2015.

16. Zhu Y, Farrehi PM and Fay WP: Plasminogen activator inhibitor type 1 enhances neointima formation after oxidative vascular injury in atherosclerosis-prone mice. Circulation 103: 3105-3110, 2001.

17. Wang J, Yan G, Guo H, Zhu Y, Shui X, He Y, Chen C and Lei W: ITE promotes hypoxia-induced transdifferentiation of human pulmonary aterial endothelial cells possibly by activating transforming growth factor- $\beta /$ Smads and MAPK/ERK pathways. J Cell Biochem 120: 19567-19577, 2019.

18. Nakamura H, Kitazawa K, Honda $H$ and Sugisaki T: Roles of and correlation between alpha-smooth muscle actin, CD44, hyaluronic acid and osteopontin in crescent formation in human glomerulonephritis. Clin Nephrol 64: 401-411, 2005.

19. Kato Y, Furusyo N, Tanaka Y, Ueyama T, Yamasaki S, Murata M and Hayashi J: The relation between serum endostatin level and carotid atherosclerosis in healthy residents of Japan: Results from the Kyushu and Okinawa Population Study (KOPS). J Atheroscler Thromb 24: 1023-1030, 2017.

20. Ruge T, Carlsson AC, Kjøller E, Hilden J, Kolmos HJ, Sajadieh A, Kastrup J, Jensen GB, Larsson A, Nowak C, et al: Circulating endostatin as a risk factor for cardiovascular events in patients with stable coronary heart disease: A CLARICOR trial sub-study. Atherosclerosis 284: 202-208, 2019.

21. Paszkowiak JJ and Dardik A: Arterial wall shear stress: Observations from the bench to the bedside. Vasc Endovascular Surg 37: 47-57, 2003.

22. Dolan JM, Meng H, Singh S, Paluch R and Kolega J: High fluid shear stress and spatial shear stress gradients affect endothelial proliferation, survival, and alignment. Ann Biomed Eng 39: 1620-1631, 2011.

23. Franck G: Role of mechanical stress and neutrophils in the pathogenesis of plaque erosion. Atherosclerosis 318: 60-69, 2021.

24. Tang R, Zhang G and Chen SY: Smooth muscle cell proangiogenic phenotype induced by cyclopentenyl cytosine promotes endothelial cell proliferation and migration. J Biol Chem 291: 26913-26921, 2016.

25. Palumbo R, Gaetano C, Antonini A, Pompilio G, Bracco E, Rönnstrand L, Heldin CH and Capogrossi MC: Different effects of high and low shear stress on platelet-derived growth factor isoform release by endothelial cells: Consequences for smooth muscle cell migration. Arterioscler Thromb Vasc Biol 22: 405-411, 2002. 
26. Slager CJ, Wentzel JJ, Gijsen FJ, Schuurbiers JC, van der Wal AC, van der Steen AF and Serruys PW: The role of shear stress in the generation of rupture-prone vulnerable plaques. Nat Clin Pract Cardiovasc Med 2: 401-407, 2005.

27. Miosge N, Simniok T, Sprysch P and Herken R: The collagen type XVIII endostatin domain is co-localized with perlecan in basement membranes in vivo. J Histochem Cytochem 51: 285-296, 2003.

28. Goyanes AM, Moldobaeva A, Marimoutou M, Varela LC, Wang L, Johnston LF, Aladdin MM, Peloquin GL, Kim BS, Damarla M, et al: Functional impact of human genetic variants of collagen 18A1/endostatin on pulmonary endothelium. Am J Respir Cell Mol Biol 62: 524-534, 2020.

29. Hutter R, Sauter BV, Reis ED, Roque M, Vorcheimer D, Carrick FE, Fallon JT, Fuster V and Badimon JJ: Decreased reendothelialization and increased neointima formation with endostatinoverexpression in a mouse model of arterial injury. Circulation 107: 1658-1663, 2003

30. O'Reilly MS, Boehm T, Shing Y, Fukai N, Vasios G, Lane WS, Flynn E, Birkhead JR, Olsen BR and Folkman J: Endostatin: An endogenous inhibitor of angiogenesis and tumor growth. Cell 88 : 277-285, 1997.

31. Dhanabal M, Ramchandran R, Waterman MJ, Lu H, Knebelmann B, Segal M and Sukhatme VP: Endostatin induces endothelial cell apoptosis. J Biol Chem 274: 11721-11726, 1999.

32. Abdollahi A, Hahnfeldt P, Maercker C, Gröne HJ, Debus J, Ansorge W, Folkman J, Hlatky L and Huber PE: Endostatin's antiangiogenic signaling network. Mol Cell 13: 649-663, 2004.

33. Kim YM, Jang JW, Lee OH, Yeon J, Choi EY, Kim KW, Lee ST and Kwon YG: Endostatin inhibits endothelial and tumor cellular invasion by blocking the activation and catalytic activity of matrix metalloproteinase. Cancer Res 60: 5410-5413, 2000.

34. Hoffmann J, Marsh LM, Pieper M, Stacher E, Ghanim B, Kovacs G, König P, Wilkens H, Haitchi HM, Hoefler G, et al Compartment-specific expression of collagens and their processing enzymes in intrapulmonary arteries of IPAH patients. Am J Physiol Lung Cell Mol Physiol 308: L1002-L1013, 2015.
35. Herman MP, Sukhova GK, Libby P, Gerdes N, Tang N, Horton DB, Kilbride M, Breitbart RE, Chun M and Schönbeck U: Expression of neutrophil collagenase (matrix metalloproteinase-8) in human atheroma: A novel collagenolytic pathway suggested by transcriptional profiling. Circulation 104: 1899-1904, 2001.

36. Ye S: Putative targeting of matrix metalloproteinase- 8 in atherosclerosis. Pharmacol Ther 147: 111-122, 2015.

37. Laxton RC, Hu Y, Duchene J, Zhang F, Zhang Z, Leung KY, Xiao Q, Scotland RS, Hodgkinson CP, Smith K, et al: A role of matrix metalloproteinase-8 in atherosclerosis. Circ Res 105: 921-929, 2009

38. Zhang F, Li S, Song J, Liu J, Cui Y and Chen H: Angiotensin-(1-7) regulates angiotensin II-induced matrix metalloproteinase- 8 in vascular smooth muscle cells. Atherosclerosis 261: 90-98, 2017.

39. Sato Y: Endostatin as a biomarker of basement membrane degradation. J Atheroscler Thromb 24: 1014-1015, 2017.

40. Spring S, van der Loo B, Krieger E, Amann-Vesti BR, Rousson V and Koppensteiner R: Decreased wall shear stress in the common carotid artery of patients with peripheral arterial disease or abdominal aortic aneurysm: Relation to blood rheology, vascular risk factors, and intima-media thickness. J Vasc Surg 43: 56-63, 2006.

41. Osswald A, Karmonik C, Anderson JR, Rengier F, Karck M, Engelke J, Kallenbach K, Kotelis D, Partovi S, Böckler D and Ruhparwar A: Elevated wall shear stress in aortic type B dissection may relate to retrograde aortic type A dissection: A computational fluid dynamics pilot study. Eur J Vasc Endovasc Surg 54: 324-330, 2017.

42. Holsti M, Wanhainen A, Lundin C, Björck M, Tegler G, Svensson J and Sund M: Circulating vascular basement membrane fragments are associated with the diameter of the abdominal aorta and their expression pattern is altered in AAA tissue. Eur J Vasc Endovasc Surg 56: 110-118, 2018.

(i) $($ ) This work is licensed under a Creative Commons Attribution-NonCommercial-NoDerivatives 4.0 International (CC BY-NC-ND 4.0) License. 